\title{
Preparation of barley and yeast $\beta$-glucan scaffolds by hydrogel foaming: Evaluation of dexamethasone release
}

\author{
Marta Salgado $^{\mathrm{a}}$, Soraya Rodríguez-Rojo ${ }^{\mathrm{a}}$, Rui L. Reis ${ }^{\mathrm{b}, \mathrm{c}}$, María José Cocero ${ }^{\mathrm{a}}$, \\ Ana Rita C. Duarte ${ }^{\mathrm{b}, \mathrm{c}, *}$ \\ ${ }^{\text {a }}$ High Pressure Processes Group, Department of Chemical Engineering and Environmental Technology, EII Sede Mergelina, University of Valladolid, 47011 Valladolid, \\ Spain \\ b 3B's Research Group-Biomaterials, Biodegradables and Biomimetics, University of Minho, Headquarters of the European Institute of Excellence on Tissue Engineering \\ and Regenerative Medicine, AvePark, 4805-017 Barco, Guimarães, Portugal \\ ' ICVS/3B's - PT Government Associate Laboratory, Braga, Guimarães, Portugal
}

\section{A R T I C L E I N F O}

\section{Keywords:}

B-Glucan

Supercritical hydrogel foaming

Dexamethasone

Scaffolds

Drug delivery

\begin{abstract}
A B S T R A C T
Porous polymeric materials are studied in tissue engineering, because they can act as support for cell proliferation and as drug delivery vehicles for regeneration of tissues. Hydrogel foaming with supercritical $\mathrm{CO}_{2}$ is a suitable alternative for the creation of these structures, since it avoids the use of organic solvents and high temperature in the processing. In this work, $\beta$-glucans were used as raw materials to create hydrogels due to their easily gelation and biological properties. The enhancement of porosity was generated by a fast decompression after keeping the hydrogels in contact with $\mathrm{CO}_{2}$. The effect of the processing conditions and type of $\beta$-glucan in the final properties was assessed regarding morphological and mechanical properties. Finally, the ability of these materials to sustainably deliver dexamethasone was evaluated. The scaffolds had good morphology and provided a controlled release, thus being suitable to be used as scaffolds and drug delivery vehicles.
\end{abstract}

\section{Introduction}

Tissue engineering involves the use of a combination of polymeric scaffolds, cells and bioactive compounds (such as growth factors) in order to mimic the host tissue and provide the necessary cues to induce its regeneration. The aim is to enhance the regeneration of damaged tissue with an implant containing stem or differentiated cells which allow in situ growth of new tissue [1,2].

The scaffolds must meet certain requirements to be used in cartilage or bone tissue engineering $[3,4]$. They have to be prepared from a biocompatible material that degrades at the same rate as the growth of the tissue, so that it disappears when new tissue is created [5]. Also they must have similar mechanical characteristics to the tissue they are going to replace, depending on whether it is a soft tissue like cartilage, or a hard and more compact tissue like bone. The mechanical properties of the polymeric scaffolds can be tuned by the addition of ceramic materials [2]. Not only the incorporation of ceramics like hydroxyapatite or $\beta$-tricalcium phosphate improves the mechanical properties but also enhances the biocompatibility and integration of the material in the implant zone [6]. Regarding the structure of the scaffold, macro pores in the order of $150 \mu \mathrm{m}$ are required. Furthermore, the pores have to be highly interconnected to allow the transport of nutrients and byproducts to and from the cells in the matrix, respectively [7]. The scaffolds should not only act as support for cellular growth, but also as drug delivery vehicle for some active compounds. For instance, they could include an anti-inflammatory agent that prevents an undesired inflammatory reaction of the surrounding tissues upon implantation [1].

$\beta$-Glucans were chosen in this work as biopolymer for the fabrication of scaffolds. Polysaccharides are widely used with this purpose because they are non-toxic, biocompatible, biodegradable and are obtained from renewable sources in nature. In this context, $\beta$-glucans are a good option, since they are able to create a hydrogel easily by thermal changes. Besides, they offer some interesting biological properties, such as the ability to improve wound healing or modulate the immune system, and their anti-inflammatory and anti-bacterial properties, that increase their potential in medical and pharmaceutical applications [8-10]. Some previous works explore the use of different $\beta$-glucans for tissue engineering. Przekora et al. studied the properties of a scaffold composed of bacterial 1,3- $\beta$-glucan (curdlan) with chitosan

\footnotetext{
* Corresponding author at: 3B's Research Group—Biomaterials, Biodegradables and Biomimetics, University of Minho, Headquarters of the European Institute of Excellence on Tissue Engineering and Regenerative Medicine, AvePark, 4805-017 Barco, Guimarães, Portugal.

E-mail address: aduarte@dep.uminho.pt (A.R.C. Duarte).
} 
and hydroxyapatite prepared by gelling of the components and airdrying the hydrogels [11]. They found out that the addition of $\beta$-glucan to hydroxyapatite increased the elasticity and water uptake capacity of the scaffold, suggesting a better adaptation into the implant site, although mechanical strength was decreased [12]. Also curdlan was effectively used for wound dressing in burn injuries [13]. Furthermore, regarding cell culture, the presence of $\beta$-glucan in the chitosanhydroxyapatite composite enhanced cell adhesion and growth [14]. Another work also reported an improvement in cell regeneration through a better adhesion and proliferation in membranes of poly (lactic-co-glycolic acid) when they were mixed with $1,3-1,6-\beta$-glucans [15]. Nevertheless, in previous works, the $\beta$-glucan-based materials for these applications were created mainly by moulding or electrospinning of the polymeric solution. Up to our knowledge, there are no reports aiming to produce $\beta$-glucan scaffolds with enhanced porous properties for tissue engineering applications, particularly in which concerns bone regeneration.

Several techniques are used to create porous scaffolds, such as salt leaching, phase separation or sintering [16-19]. However, these procedures imply the introduction of organic solvents or very high temperatures, and further purification steps to completely remove toxic residues [20]. Foaming of polymers with supercritical $\mathrm{CO}_{2}$ is an extensively used alternative, since the porosity is produced using $\mathrm{CO}_{2}$ as blowing agent, and it is easily removed later by decompression $[3,21]$. This process is based on the ability of supercritical $\mathrm{CO}_{2}$ to plasticize and saturate the polymer above its critical point. When pressure is released, supersaturation of the $\mathrm{CO}_{2}$ occurs, giving rise to bubble nuclei inside the polymer that result in pores upon solidification [22]. Nevertheless, this procedure can only be applied to amorphous or semi-crystalline hydrophobic polymers, but not to hydrophilic polymers that do not experiment any phase transition before thermal degradation $[23,24]$. In these cases, porous structures can be formed with supercritical $\mathrm{CO}_{2}$ by developing first a hydrogel so that the dense gas is dissolved in the aqueous phase and is also able to penetrate and swell the rubbery structure of the hydrogel [24]. Then, when the system is depressurize, pores are created by bubble formation due to supersaturation of $\mathrm{CO}_{2}$. With this process, highly interconnected structures from natural-origin polymers can be prepared. Different studies reported in the literature show the ability to foam natural-based polymers using this technique, particularly chitosan, elastin and collagen $[23,25,26]$.

In this work, porous scaffolds were prepared in order to be used as polymeric matrix for bone regeneration. The scaffolds were created by hydrogel foaming with supercritical $\mathrm{CO}_{2}$ with 2 different $\beta$-glucans: $(1-3,1-4)-\beta$-glucans from barley and $(1-3,1-6)-\beta$-glucans from yeast Saccharomyces cerevisae. Both $\beta$-glucans differ in their structure and properties: whereas barley $\beta$-glucan is a linear, water soluble polymer, yeast $\beta$-glucan has a branched conformation and is not soluble in water. Thus, their behavior is different and they have to be processed in a different way in order to create the hydrogel. Also, this will influence the structure and the properties of the final products, which were analyzed regarding morphology and mechanical properties, and compared to freeze-dried materials as control. Besides, dexamethasone was included in the formulations as a model active compound to study the release from the scaffold. Dexamethasone was chosen as model active compound because it promotes the differentiation of stem cells towards osteogenic lineage and, at the same time, has anti-inflammatory effect in the implant zone $[27,28]$.

\section{Materials and methods}

\subsection{Materials}

Barley $\beta$-glucans (BBG, 75\% purity; Glucagel, kindly supplied by DKSH, France) and yeast $\beta$-glucans (YBG, 64\% purity; L-Naturae Nutraceutical, kindly supplied by Naturae, Spain) were used as raw materials to create the hydrogels. Dexamethasone (CAS 50-02-2) was purchased from Sigma. PBS was prepared from tablets (Sigma).

\subsection{Production of $\beta$-glucan hydrogels}

BBG hydrogels $(4 \% \mathrm{w} / \mathrm{w})$ were produced by dissolving the $\beta$ glucans in water at $80^{\circ} \mathrm{C}$, boiling them for $5 \mathrm{~min}$ and then keeping them at $75^{\circ} \mathrm{C}$ for $1 \mathrm{~h}$. After that time, the solution was poured into 96well plate molds and kept at $4{ }^{\circ} \mathrm{C}$ for 3 days until they became gel.

YBG hydrogels $(2.5 \% \mathrm{w} / \mathrm{w})$ were created from a suspension. YBG was first stirred for $30 \mathrm{~min}$, in order to correctly hydrate them and achieve a homogeneous dispersion. Then they were heated at $90{ }^{\circ} \mathrm{C}$ for $1 \mathrm{~h}$ and poured into molds, where they were kept overnight to form the hydrogel. The concentration of both $\beta$-glucans was chosen as the lowest which allowed the formation of the hydrogel.

\subsection{Hydrogel foaming with supercritical $\mathrm{CO}_{2}$}

The hydrogels were placed in a high-pressure vessel, preheated at $37^{\circ} \mathrm{C}$. Then the vessel was closed and $\mathrm{CO}_{2}$ was fed up to the chosen operating pressure, namely $8,12,16$ or $20 \mathrm{MPa}$, using a high-pressure pump (Haskell, MCPV-71). The hydrogels were kept in contact with supercritical $\mathrm{CO}_{2}$ for $1.5 \mathrm{~h}$, in order to have enough time to allow the diffusion of $\mathrm{CO}_{2}$ into the bulk of the hydrogels and saturate them. After that time, a fast decompression was performed to promote the foaming and at the same time freezing off the structures. In this way, the porous structure produced was stabilized and kept overnight at $-80{ }^{\circ} \mathrm{C}$. Water was removed by freeze-drying $\left(-80^{\circ} \mathrm{C}\right.$, vacuum lower than $\left.0.5 \mathrm{mbar}\right)$. As a control, hydrogel samples not subjected to pressurized carbon dioxide were directly frozen and freeze-dried.

\subsection{Morphological characterization of the scaffolds}

Micro-Computed Tomography (Micro-CT) was used to evaluate the porosity and pore size of the 3D structures obtained. The images were acquired on a high-resolution micro-CT SkyScan 1272 scanner (Bruker, Germany) using a voltage of $50 \mathrm{kV}$ and a current of $240 \mu \mathrm{A}$. Images were acquired with an exposure time of $160 \mathrm{~ms}$ and a pixel size of $15.99 \mu \mathrm{m}$. After image acquisition the noise was reduced with nRecon software. CT Analyser software (SkyScan, Belgium) was used to obtain representative data sets of the samples and converting them into $2 \mathrm{D}$ images. For each set of conditions, 3 different samples were analyzed. The interconnectivity of the scaffold is calculated according to the formula: $I=\left[\left(V_{\text {totalpores }}-V_{\text {disconnectedpores }}\right) / V_{\text {totalpores }}\right] \times 100$, where the volume of the disconnected pores is defined to be the volume of porous which are not connected by channels higher than $50 \mu \mathrm{m}$.

The produced scaffolds were observed by scanning electron microscopy (SEM) with a high-resolution field emission scanning electron microscope with focus ion beam (Auriga Compact, Zeiss). The samples were cut in liquid nitrogen and the sections were placed by mutual conductive adhesive tape on aluminum holders and covered with gold palladium using a sputter coater.

\subsection{Mechanical properties}

The mechanical behavior of the samples was assessed in compression mode using a universal testing machine (Instron 5540). The scaffolds were compressed at $1 \mathrm{~mm} \mathrm{~min}^{-1}$ until a maximum deformation of $70 \%$ of the initial height. The compressive Young modulus was determined as the initial slope in the stress-strain graphs. The analysis was performed in triplicate.

\subsection{In vitro release of dexamethasone}

Dexamethasone was loaded in the initial aqueous solution of $\beta$ glucans at different concentrations, namely 5 and $10 \mathrm{wt} \%$ in respect to 

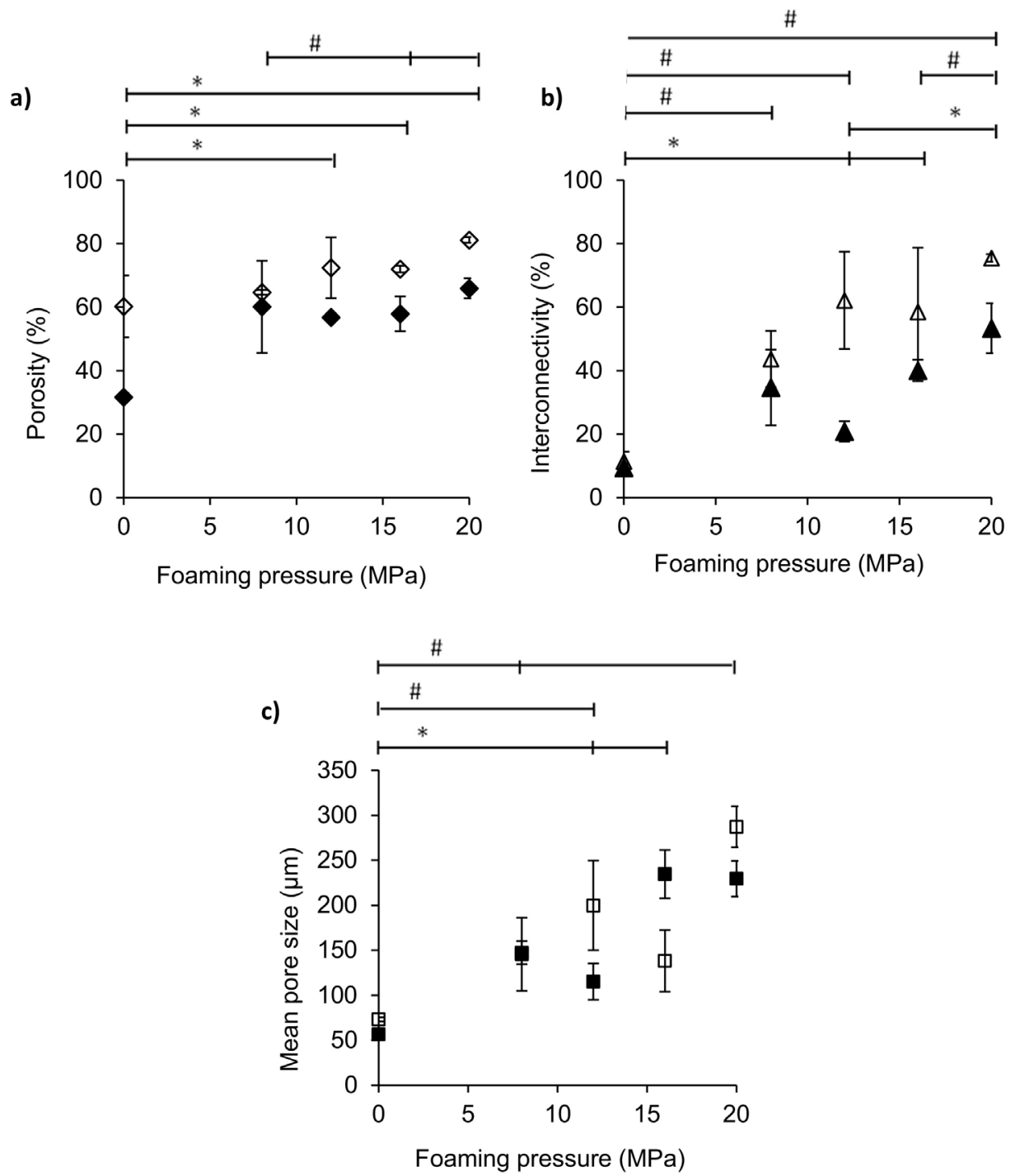

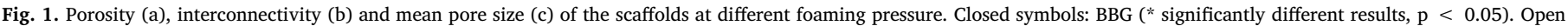
symbols: YBG (\# significantly different results, $\mathrm{p}<0.05$ ).

the weight of the polymer, and then the processing of the structures was done as described before (Sections 2.2 and 2.3). The conditions selected for the foaming were $37^{\circ} \mathrm{C}$ and $12 \mathrm{MPa}$.

The different scaffolds were immersed in $20 \mathrm{~mL}$ PBS in a water bath at $37^{\circ} \mathrm{C}$. Aliquots of $150 \mu \mathrm{L}$ of the liquid medium were withdrawn at different time points $(5,10,15$ and $30 \mathrm{~min}, 1,2,3,4.5,6,8,24$ and $32 \mathrm{~h}$, and daily from 2 to 11 days) and replaced with the same quantity of fresh PBS. The samples were analyzed by UV-vis spectrophotometry at $245 \mathrm{~nm}$ and the absorbance value was adjusted into a calibration curve between 0 and $0.05 \mathrm{~g} / \mathrm{L}$ of dexamethasone to determine the drug concentration released into the liquid. Three different samples were prepared for each time point. The replacement of liquid with fresh PBS was taken into account in the calculations of the cumulative mass of dexamethasone.

The release kinetics was analyzed with different empirical models in order to determine which one better represented the release behavior from the structures, in particular Korsmeyer-Peppas (Eq. (1)), zero order (Eq. (2)), first order (Eq. (3)), Higuchi (Eq. (4)) and HixsonCrowell's models (Eq. (5)) [29].

$\frac{M_{t}}{M_{\infty}}=k t^{n}$
$\frac{M_{t}}{M_{\infty}}=k t$

$\frac{M_{t}}{M_{\infty}}=e^{-k t}$

$\frac{M_{t}}{M_{\infty}}=k t^{1 / 2}$

$\left(M_{\infty}\right)^{1 / 3}-\left(M_{r}\right)^{1 / 3}=k t$

In these equations, $M_{t}$ is the quantity of drug released at time $t, M_{r}$ is the quantity of mass retained at time $t, M_{\infty}$ is defined as the quantity of drug that would be released at infinite time, which is the total quantity of drug initially on the scaffold and $\mathrm{k}$ is the kinetic constant.

In order to complete the information from the Korsmeyer-Peppas model, Peppas and Sahlin proposed another empirical equation to determine the contribution of each release mechanism (diffusion controlled or swelling controlled) to the total drug release [30]:

$\frac{M_{t}}{M_{\infty}}=k_{1} t^{m}+k_{2} t^{2 m}$

Where $\mathrm{m}$ is the diffusion exponent of the pure Fickian diffusion, which is 0.45 for a cylindrical geometry. In this equation, the first term of the right $\left(\mathrm{k}_{1} \mathrm{t}^{\mathrm{m}}\right)$ corresponds to the Fickian contribution, and the second 
$4 \%$ BBG

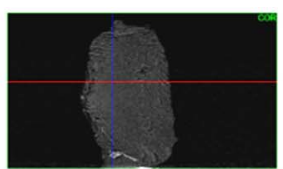

a)
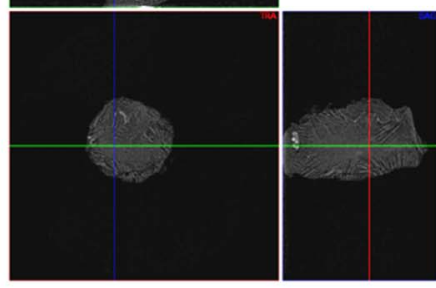

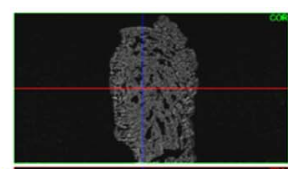

b)

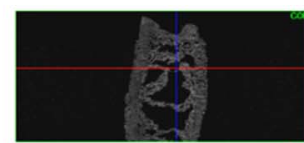

c)
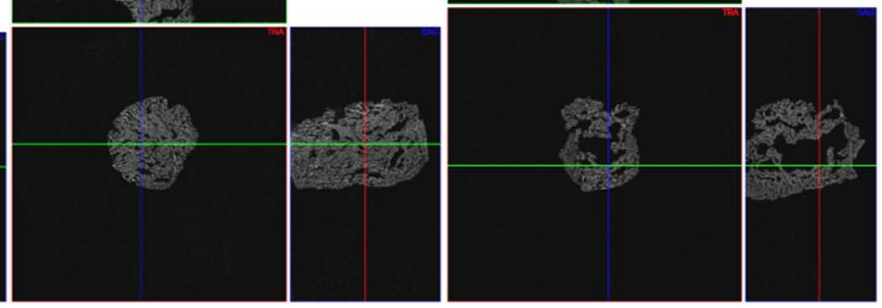
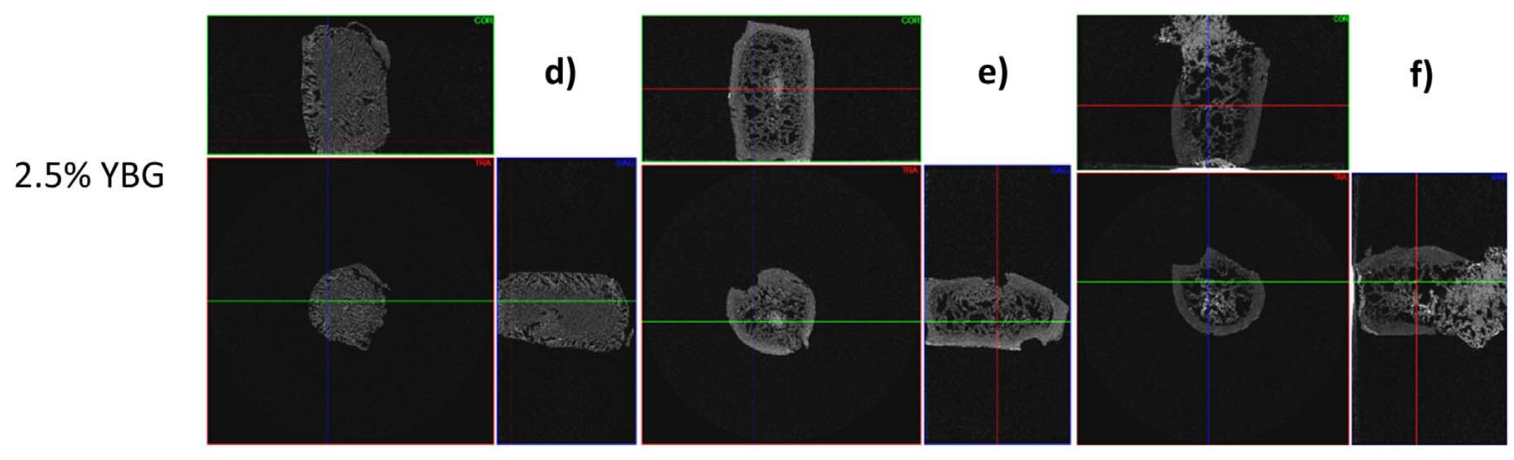

Fig. 2. Micro-ct reconstructions of $\beta$-glucan scaffolds produced by freeze-drying (a and d) and by foaming with $\mathrm{CO}_{2}$ at 12 (b and e) and $20 \mathrm{MPa}$ (c and f).

$\left(\mathrm{k}_{2} \mathrm{t}^{2 \mathrm{~m}}\right)$ corresponds to the relaxation contribution. By fitting $\mathrm{k}_{1}$ and $\mathrm{k}_{2}$ to the experimental data up to $60 \%$ release, it is possible to calculate the relative contribution of Fickian (F) and relaxation (R) mechanisms as follows:

$F=\frac{1}{1+\frac{k_{2}}{k_{1}} t^{m}}$

$\frac{R}{F}=\frac{k_{2}}{k_{1}} t^{m}$

\subsection{Statistical analysis}

For the morphological analysis, the average values and standard deviation of porosity, mean pore size and interconnectivity were evaluated, and Student's $t$-test was performed (unpaired samples, unequal variances) in order to check whether there were significant differences between each sample for each parameter, with a significance p-value of 0.05 .

Statistical differences between the calculated Young moduli and maximum stress in compression test were evaluated by Student's $t$-test for each $\beta$-glucan (unpaired samples, unequal variances, significance pvalue of 0.05$)$.

\section{Results and discussion}

\subsection{Morphological characterization of $\beta$-glucan scaffolds}

$\beta$-Glucan scaffolds with enhanced porous properties were obtained by hydrogel foaming with supercritical $\mathrm{CO}_{2}$. An important step in the hydrogel foaming is the stabilization of the structures. The JouleThompson effect plays a key role in this process. Upon the fast decompression, the samples were frozen due to the sudden expansion of $\mathrm{CO}_{2}$ to atmospheric pressure, so that the bigger the pressure drop, the greater the cooling effect and hence stabilization of the materials. Thus, the samples were completely frozen when working at $12 \mathrm{MPa}$ or more, but at $8 \mathrm{MPa}$ it was more difficult to stabilize the structures and this resulted in less homogeneity.

Morphological properties were obtained by micro-CT (Fig. 1). Porosity, pore size and interconnectivity followed an increasing tendency with the foaming pressure for both $\beta$-glucans, although some differences were observed between them. For BBG, porosity increased from $30 \%$ when the material was just freeze-dried to $55-60 \%$ when the structures were foamed with supercritical $\mathrm{CO}_{2}$, without significant differences with the pressure (Fig. 1a). On the contrary, YBG had higher porosity than BBG when it was processed just by freeze-drying (60\%), which could be due to the lower concentration of polymer used to create the hydrogel. When the material was foamed, the porosity increased up to $80 \%$ with $20 \mathrm{MPa}$. These values are in the range of those of natural bone, which is between 50 and $90 \%$ porosity [31].

Regarding interconnectivity of the pores, YBG showed a greater increase with the foaming pressure when compared with BBG (Fig. 1b). Although interconnectivity was the same for both $\beta$-glucans obtained by freeze-drying (around 10\%), after supercritical foaming with $\mathrm{CO}_{2}$ it was bigger for YBG in all the range of pressure tested, reaching 53\% for BBG and $75 \%$ for YBG at $20 \mathrm{MPa}$. Nevertheless, there was a noticeable increase for both polymers when they were processed with supercritical $\mathrm{CO}_{2}$, compared to conventional freeze-drying.

Despite the differences between BBG and YBG in porosity and interconnectivity, both $\beta$-glucans had very similar mean pore size, and the same increasing tendency with foaming pressure, from 50 to $75 \mu \mathrm{m}$ by freeze-drying, to $230-290 \mu \mathrm{m}$ at $20 \mathrm{MPa}$ (Fig. 1c). This increment in pore size makes the scaffolds suitable to be used in bone regeneration regarding pore size, since $100 \mu \mathrm{m}$ is considered to be the minimum size required for proper bone growth. In addition, pore size up to $300 \mu \mathrm{m}$ contributes to the formation of capillaries that enhance vascularization of the new tissue [31]. This effect of foaming pressure in pore size was not observed and had not been described in previous works reporting foaming of chitosan and chitin hydrogels $[23,26]$.

At higher foaming pressure, more quantity of $\mathrm{CO}_{2}$ saturates the hydrogel [32]. Thus, more nuclei are formed during depressurization, and each one will correspond later to one pore. This partially explains the increase in porosity with the foaming pressure. However, at higher pressures, the amount of $\mathrm{CO}_{2}$ in the hydrogels is too high and it cannot 
be totally released during the decompression. Thus dry ice is formed inside the matrix, which will then be sublimated when the freeze-drying takes place [26]. In this way, it creates channels that link the pores, producing at the same time an increase in the porosity and in interconnectivity. Finally, coalescence of pores and channels generates the increase in pore size.

The increase in porosity and pore size is easily observed on the 2D images of the micro-CT reconstructions of the materials (Fig. 2). By freeze-drying (Fig. 2a and d), the samples presented less void spaces, and only the typical channels correspondent to water crystals were noticed. A much porous structure was obtained by foaming with supercritical $\mathrm{CO}_{2}$ (Fig. 2b and e), with the pores well-distributed along the material. However, when the pressure was increased up to $20 \mathrm{MPa}$ (Fig. 2c and f), non-homogeneous samples with very large pores were produced, so that the material was almost hollow on the inside and the polymer being placed mostly on the outer part. According to those results, pressures of 12 or $16 \mathrm{MPa}$ can be identified as the optimum operating conditions in order to establish a balance between the pore size, porosity and interconnectivity required for tissue engineering applications and the homogeneity of the samples produced.

The structure of the scaffolds was also analyzed by SEM (Fig. 3). In the freeze-dried samples not foamed, the pores were smaller, and were oriented following the directions of the crystallization of ice. On the contrary, when the samples were foamed, the pores increased. As it was observed with micro-CT images, the most homogeneous porosity was observed for the samples processed at intermediate pressures (12 and $16 \mathrm{MPa}$ ). At 8 and $20 \mathrm{MPa}$, a combination of small and very large pores was noticed. In the case of $8 \mathrm{MPa}$, this fact is due to the incomplete freezing of the material upon decompression, which made the structures very unstable and the porosity could not be well maintained. At $20 \mathrm{MPa}$, as it was aforementioned, the presence of big pores was a consequence of the joining of the smaller ones because of an excess of $\mathrm{CO}_{2}$ dissolved in the hydrogel during processing. Due to the inhomogeneity of the samples produced by foaming at $20 \mathrm{MPa}$, they were disregarded for further mechanical analysis.

\subsection{Mechanical properties}

The mechanical properties of the scaffolds are highly dependent on the porosity and interconnectivity of the pores. However, the variations observed in the porous properties were not reflected in the mechanical properties. For each $\beta$-glucan, there were no significant differences with the foaming pressure in Young modulus values. For maximum stress until failure, only the value corresponding to $16 \mathrm{MPa}$ for BBG is significantly lower than the ones at lower foaming pressure. YBG samples were much more brittle and fragile, probably due to the lower polymer concentration and some of them could not even be handled and were therefore not analyzed. Fig. 4 shows the results of the compression test.

The values obtained were much lower than those of compact and spongy bone (15-20 GPa and $0.1-5 \mathrm{GPa}$, respectively) or cartilage $[12,33,34]$. Nevertheless, it has been reported that, although cartilage has values of Young modulus between $0.5-1 \mathrm{MPa}$, values around $4 \mathrm{kPa}$ enhance the formation of cartilage, whereas Young modulus higher than $40 \mathrm{kPa}$ facilitates osteogenesis [35]. Young modulus results were in the range of those obtained by other authors in elastin hydrogels (1.9 kPa, and $11.8 \mathrm{kPa}$ in a composite with tropoelastin) foamed with high-pressure $\mathrm{CO}_{2}$ [36], and slighlty lower than the ones reported for chitosan hydrogels foamed with high-pressure $\mathrm{CO}_{2}(41.6 \mathrm{kPa}$ when it was cross-linked with glutaraldehyde and $73.9 \mathrm{kPa}$ when it was crosslinked with genipin) [23]. These higher values compared to $\beta$-glucan foamed samples could be attributed to the stronger network created by chemical cross-linking rather than by thermal gelling. However, as mentioned before, porosity, pore size and interconnectivity were suitable for the scaffold to be able to host cells and allow their growth. One of the solutions to overcome this, and in order to balance porous and mechanical properties, is to load a ceramic material to the initial $\beta$ glucan solution, so that after processing by hydrogel foaming a similar porous structure would be obtained, but with enhanced mechanical properties $[37,38]$.

\subsection{In vitro release of dexamethasone}

The most promising scaffolds for tissue engineering were obtained with $4 \% \mathrm{BBG}$ prepared at $37{ }^{\circ} \mathrm{C}$ and $12 \mathrm{MPa}$, hence this formulation was the one chosen to proceed with the impregnation of dexamethasone and the study of these matrices as possible drug delivery systems. Following previous results reported in the literature, two different concentrations of dexamethasone were loaded in the scaffolds, 5 and $10 \mathrm{wt} \%[39,40]$. We observed that the release profile of dexamethasone into PBS (Fig. 5)
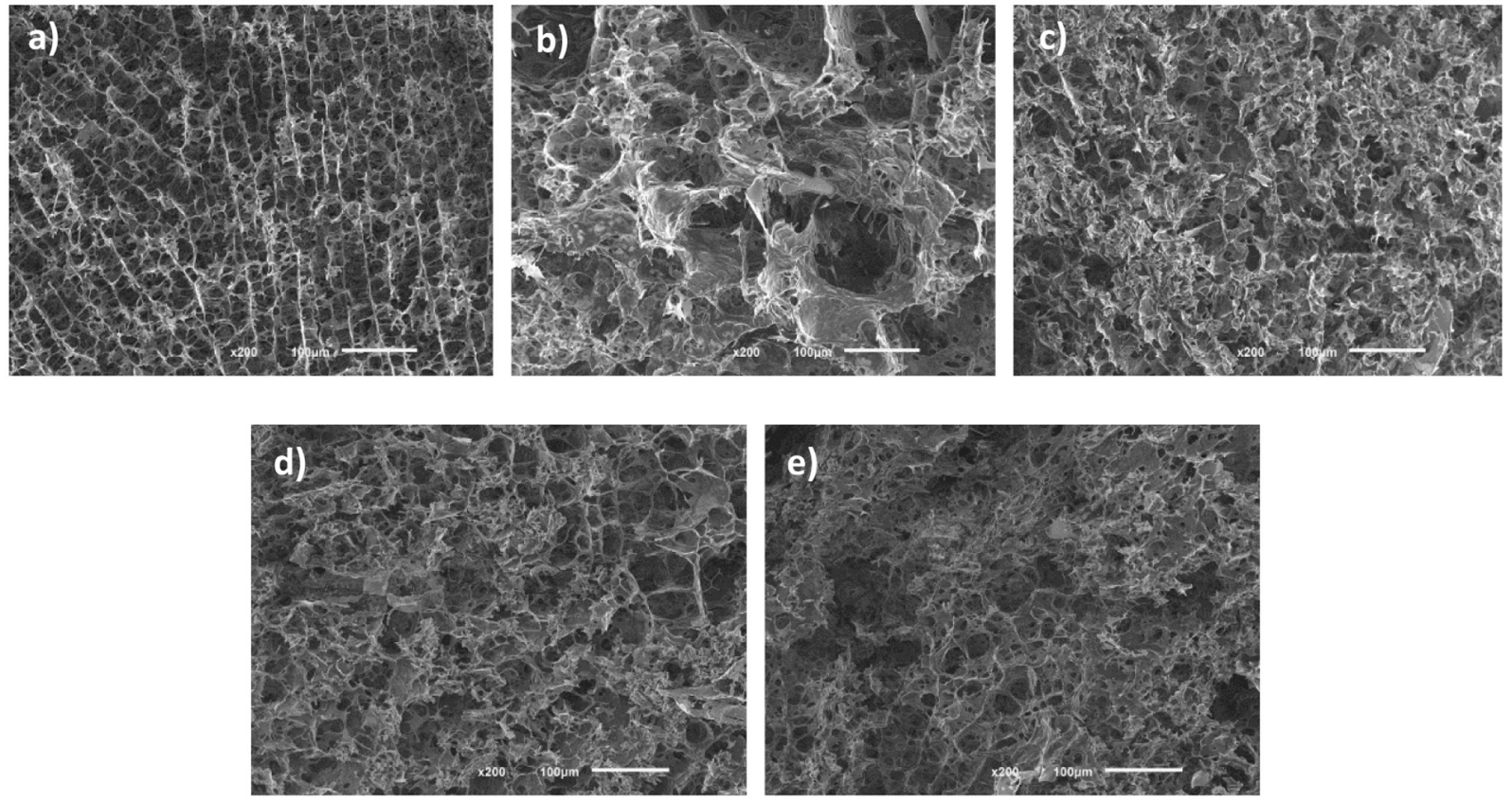

Fig. 3. SEM images of $4 \%$ BBG scaffolds produced by freeze-drying (a) and by foaming with $\mathrm{CO}_{2}$ at 8 (b), 12 (c), 16 (d) and $20 \mathrm{MPa}$ (e). Scale bar: $100 \mu \mathrm{m}$. Magnification: $\times 200$. 


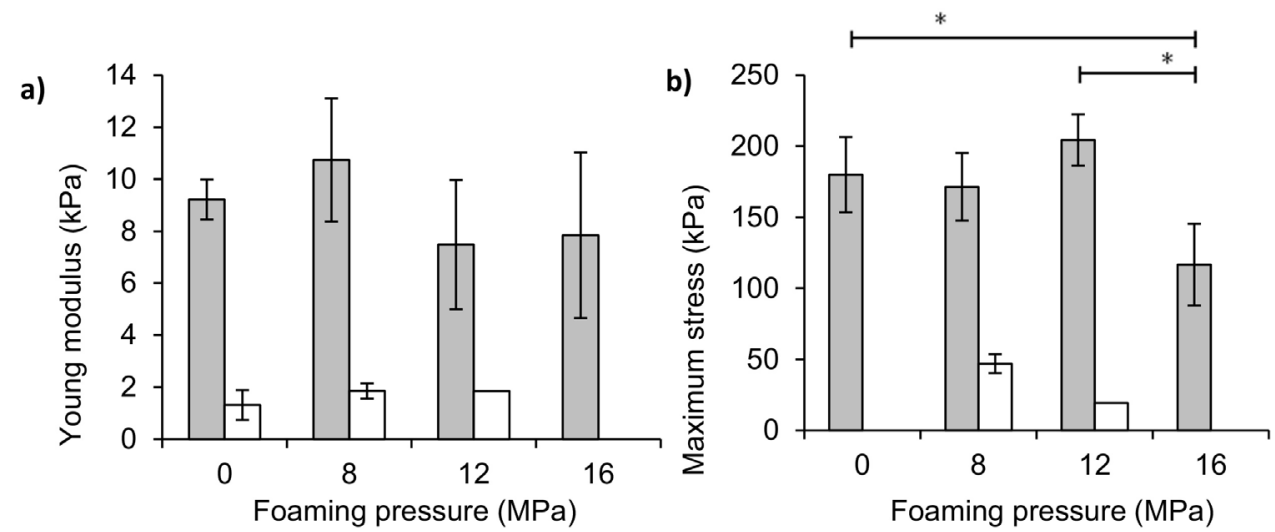

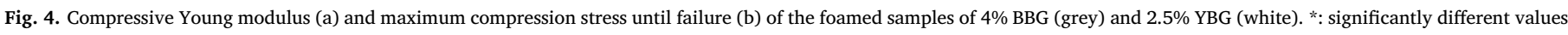
for BBG samples ( $\mathrm{p}<0.05)$.

was very similar for both concentrations of the active compound tested. This would be expected since it depends mostly on the properties of the polymeric matrix and on the interactions between the active compound and the polymer. First, there was a burst release up to $8 \mathrm{~h}$, in which 36 and $44 \mathrm{mg}$ of dexamethasone per $\mathrm{g}$ of scaffold was released to the liquid medium for each concentration (which accounted for 75 and $49 \%$ of the total loaded amount, respectively). After that period, a slower release was obtained until 4 days. At that point, the release reached a constant value and was maintained for the following days, around 51 and $78 \mathrm{mg}$ dex/g scaffold for 5 and $10 \%$ dexamethasone, respectively. Much faster release of dexamethasone $(90 \%$ in $2 \mathrm{~h}$ ) was obtained in chitosan scaffolds loaded by supercritical impregnation of the freeze-dried material [41]. In chitosan nanoparticles prepared by mixing dexamethasone in the initial solution and then freeze-drying of the particles, a release profile similar to ours was obtained [42].

The initial burst release is usually attributed to the active compound present on the surface of the materials. The fast release up to $8 \mathrm{~h}$ achieved in our work could be a consequence of a combination of the release of the superficial drug and the highly porous matrix, which allowed a rapid penetration of PBS into the scaffold and the extraction of dexamethasone present also in the surface of the more accessible pores towards the liquid medium during the first hours. Afterwards, a sustained release was observed for the following days due to the diffusion of the drug from the polymeric matrix to the liquid medium. A initial release rate similar to ours (around 30-35 mg dexamethasone/ $\mathrm{g}$ scaffold in the first $8 \mathrm{~h}$ ) was observed in a blend of starch and polylactic acid scaffold produced by supercritical-assisted phase inversion, although after that initial period the release was slower and controlled up to 21 days [39]. This longer release period could be attributed to the higher hydrophobicity of poly-lactic acid in comparison with $\beta$-glucan, which slows down the extraction of dexamethasone by PBS.

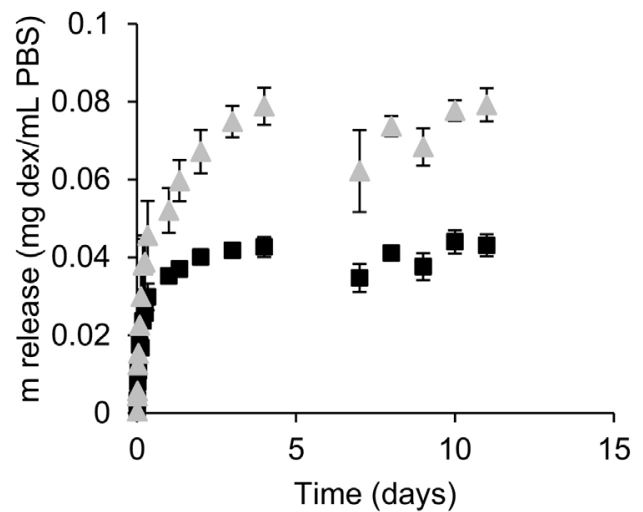

In order to be able to draw conclusions on the mechanisms governing drug release from the scaffolds, different empirical equations were applied to the experimental data. Results of the fitting of the results of release in PBS (Table 1) revealed that release for $10 \%$ dexamethasone could be well described by first-order model, which represents a control by both diffusion and relaxation of the matrix. However, in the case of $5 \%$ dexamethasone, the adjustment with Higuchi model was better. Thus, the release was mostly controlled by the diffusion of dexamethasone from the polymer to the liquid.

With Korsmeyer-Peppas' model, it is also possible to obtain the diffusion exponent, $\mathrm{n}$, which characterizes the mechanism governing the drug release [29]. In both cases the diffusion exponent determined for the BBG scaffolds loaded with dexamethasone fell in the values determined for anomalous transport for a cylindrical matrix, i.e., a value of $\mathrm{n}$ between 0.45 and 0.89 . This means that the release is governed by both diffusion and swelling of the scaffold.

In order to determine the contribution of each mechanism to the total drug release, the release data were introduced in Eq. (6), and values of $\mathrm{k}_{1}=0.0328 \mathrm{~min}^{-0.45}$ and $\mathrm{k}_{2}=0.0008 \mathrm{~min}^{-0.45}$ for $5 \%$ dexamethasone, and $\mathrm{k}_{1}=0.0277 \mathrm{~min}^{-0.45}$ and $\mathrm{k}_{2}=0.0006 \mathrm{~min}^{-0.45}$ for $10 \%$ dexamethasone were obtained. Introducing these values in Eqs. (7) and (8), it was observed that the release was mainly controlled by the diffusion of dexamethasone from the $\beta$-glucan matrix to the liquid medium (Fig. 6). At initial time, release was almost totally controlled by Fickian diffusion, although this contribution decreased with time, while relaxation of the matrix gained importance in controlling the release. Nevertheless, the contribution of the relaxation of the polymer was lower than $25 \%$. As it would be expected, the results were similar for both concentrations of dexamethasone, since the matrix was the same.

These calculations further confirm the observations from the release profiles. According to this, the release rate of dexamethasone was first

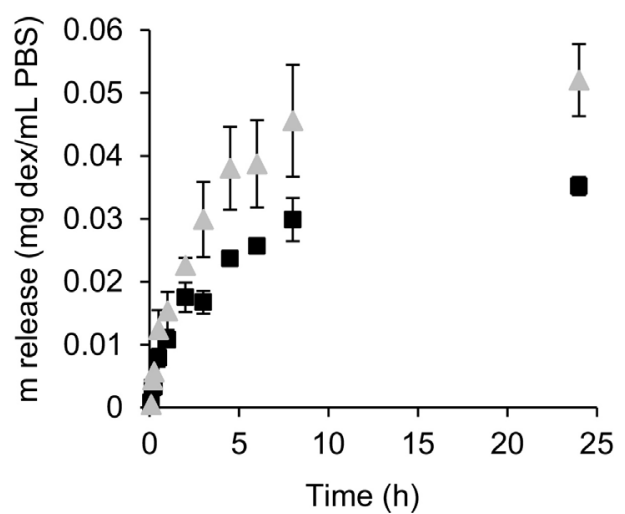

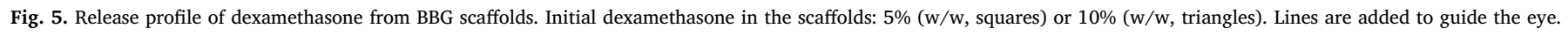


Table 1

Fitting of the release of dexamethasone to different models.

\begin{tabular}{lllllll}
\hline & \multicolumn{1}{l}{$\mathrm{r}^{2}$} & & & \\
\cline { 2 - 5 } & Zero-order & First-order & Higuchi & $\begin{array}{l}\text { Hixson- } \\
\text { Crowell }\end{array}$ & $\begin{array}{l}\text { Korsmeyer- } \\
\text { Peppas }\end{array}$ & $\mathrm{n}$ \\
\hline $5 \%$ & 0.6716 & 0.9218 & 0.9718 & 0.8464 & 0.9317 & 0.745 \\
$10 \%$ & 0.7353 & 0.9187 & 0.8932 & 0.8645 & 0.8330 & 0.721 \\
\hline
\end{tabular}

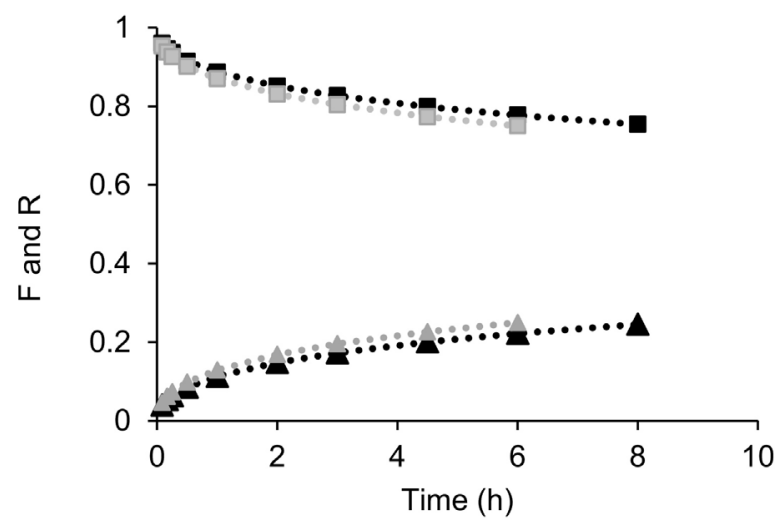

Fig. 6. Contribution of diffusion (F, squares) and relaxation of the polymer (R, triangles) to the control of the release of dexamethasone. Grey: $5 \%$, and black: $10 \%$ dexamethasone.

controlled by the diffusion rate of the drug from the scaffold to the liquid. With time, the scaffold was wetted and relaxation of chains occurred, so that this fact also contributed to the release of dexamethasone from the zones that were initially inaccessible to the liquid. However, the release mechanism governing the process was always the diffusion, possibly due to the high porosity of the scaffolds, which allowed the liquid to easily penetrate into the matrix, be in contact with the drug and extract it to the liquid medium.

\section{Conclusions}

In this work, we successfully obtained highly porous $\beta$-glucan scaffolds after supercritical foaming of the hydrogels. This process is based on the solubility of high-pressure $\mathrm{CO}_{2}$ in the water present in the hydrogels, thus the porosity is created due to supersaturation of $\mathrm{CO}_{2}$ upon decompression. Barley and yeast $\beta$-glucans were used as biopolymers for the production of the scaffolds. Influence of the foaming pressure was noticeable on the porous properties of the materials through an increase of porosity, pore size and interconnectivity with the pressure. However no significant changes were observed on their mechanical properties under compression stress. Regarding the type of $\beta$-glucan, despite the scaffolds with YBG had greater porosity and interconnectivity, mean pore size was similar for both $\beta$-glucans. Due to the differences in porosity, YBG scaffolds had lower resistance to compression. Although the morphological properties achieved in the scaffolds with both $\beta$-glucans were appropriate for support of cells and growth of new tissue, they were brittle and did not reach mechanical properties of bone or cartilage tissue by themselves. A controlled release of dexamethasone from BBG scaffolds was achieved, higher during the first $8 \mathrm{~h}$ (between 50 and 75\%), and slower until complete release in 4 days. The release was mainly controlled by diffusion of the active compound, although there was also some contribution due to relaxation of the polymeric matrix.

\section{Acknowledgements}

Authors acknowledge Ministerio de Economía y Competitividad (MINECO) through project CTQ2013-44143-R and project PIP 063/
147181 from Fundación General of the University of Valladolid for financial support. M. Salgado thanks to Ministerio de Educación, Ciencia y Deporte (MECD) for her FPU and mobility grants. S. Rodríguez-Rojo acknowledges to MINECO and UVa for her Juan de la Cierva fellowship (JCI-2012-14992). The research leading to these results has received funding from the European Union Seventh Framework Programme (FP7/2007-2013) under grant agreement number REGPOT-CT2012-316331-POLARIS and from the project "Novel smart and biomimetic materials for innovative regenerative medicine approaches" RL1 - ABMR - NORTE-01-0124-FEDER-000016) cofinanced by North Portugal Regional Operational Programme (ON.2-O Novo Norte), under the National Strategic Reference Framework (NSRF), through the European Regional Development Fund (ERDF).

\section{References}

[1] A.R.C. Duarte, J.F. Mano, R.L. Reis, Supercritical fluids in biomedical and tissue engineering applications: a review, Int. Mater. Rev. 54 (2009) 214-222.

[2] F.J. O'Brien, Biomaterials \& scaffolds for tissue engineering, Mater. Today 14 (2011) 88-95.

[3] C.A. García-González, A. Concheiro, C. Alvarez-Lorenzo, Processing of materials for regenerative medicine using supercritical fluid technology, Bioconj. Chem. 26 (2015) 1159-1171.

[4] A.R.C. Duarte, V.E. Santo, A. Alves, S.S. Silva, J. Moreira-Silva, T.H. Silva, A.P. Marques, R.A. Sousa, M.E. Gomes, J.F. Mano, R.L. Reis, Unleashing the potential of supercritical fluids for polymer processing in tissue engineering and regenerative medicine, J. Supercrit. Fluids 79 (2013) 177-185.

[5] Y. Liu, J. Lim, S.-H. Teoh, Review: development of clinically relevant scaffolds for vascularised bone tissue engineering, Biotechnol. Adv. 31 (2013) 688-705.

[6] E. Sharifi, M. Azami, A.-M. Kajbafzadeh, F. Moztarzadeh, R. Faridi-Majidi, A. Shamousi, R. Karimi, J. Ai, Preparation of a biomimetic composite scaffold from gelatin/collagen and bioactive glass fibers for bone tissue engineering, Mater. Sci. Eng. C 59 (2016) 533-541.

[7] M. Okamoto, B. John, Synthetic biopolymer nanocomposites for tissue engineering scaffolds, Prog. Polym. Sci. 38 (2013) 1487-1503.

[8] D.B. Zeković, S. Kwiatkowski, M.M. Vrvić, D. Jakovljević, C.A. Moran, Natural and modified $(1 \rightarrow 3)-\beta$-D-glucans in health promotion and disease alleviation, Crit. Rev. Biotechnol. 25 (2005) 205-230.

[9] J.A. Bohn, J.N. BeMiller, ( $\rightarrow 3$ )-B-d-Glucans as biological response modifiers: a review of structure-functional activity relationships, Carbohydr. Polym. 28 (1995) $3-14$.

[10] K. Kofuji, Y. Huang, K. Tsubaki, F. Kokido, K. Nishikawa, T. Isobe, Y. Murata, Preparation and evaluation of a novel wound dressing sheet comprised of $\beta$ glucan-chitosan complex, React. Funct. Polym. 70 (2010) 784-789.

[11] A. Przekora, K. Palka, G. Ginalska, Biomedical potential of chitosan/HA and chitosan/ß-1, 3-glucan/HA biomaterials as scaffolds for bone regeneration - a comparative study, Mater. Sci. Eng. C 58 (2016) 891-899.

[12] A. Belcarz, G. Ginalska, T. Pycka, A. Zima, A. Ślósarczyk, I. Polkowska, Z. Paszkiewicz, W. Piekarczyk, Application of $\beta-1,3-$ glucan in production of ceramics-based elastic composite for bone repair, Cent. Eur. J. Biol. 8 (2013) 534-548.

[13] S.J. Delatte, J. Evans, A. Hebra, W. Adamson, H.B. Othersen, E.P. Tagge, Effectiveness of beta-glucan collagen for treatment of partial-thickness burns in children, J. Pediatr. Surg. 36 (2001) 113-118.

[14] A. Przekora, G. Ginalska, Addition of 1, 3- $\beta$-d-glucan to chitosan-based composites enhances osteoblast adhesion, growth, and proliferation, Int. J. Biol. Macromol. 70 (2014) 474-481.

[15] H.-L. Kim, J.-H. Lee, M.H. Lee, B.J. Kwon, J.-C. Park, Evaluation of electrospun (1, 3)-(1, 6)- $\beta$-D-glucans/biodegradable polymer as artificial skin for full-thickness wound healing, Tissue Eng. Part A 18 (2012) 2315-2322.

[16] J.J. Yoon, J.H. Kim, T.G. Park, Dexamethasone-releasing biodegradable polymer scaffolds fabricated by a gas-foaming/salt-leaching method, Biomaterials 24 (2003) 2323-2329.

[17] B. Duan, M. Wang, W.Y. Zhou, W.L. Cheung, Z.Y. Li, W.W. Lu, Three-dimensional nanocomposite scaffolds fabricated via selective laser sintering for bone tissue engineering, Acta Biomater. 6 (2010) 4495-4505.

[18] M. Alizadeh, F. Abbasi, A.B. Khoshfetrat, H. Ghaleh, Microstructure and characteristic properties of gelatin/chitosan scaffold prepared by a combined freezedrying/leaching method, Mater. Sci. Eng. C 33 (2013) 3958-3967.

[19] H. Zhang, X. Liu, M. Yang, L. Zhu, Silk fibroin/sodium alginate composite nanofibrous scaffold prepared through thermally induced phase-separation (TIPS) method for biomedical applications, Mater. Sci. Eng. C 55 (2015) 8-13.

[20] H. Tai, M.L. Mather, D. Howard, W. Wang, L.J. White, J.A. Crowe, S.P. Morgan, A. Chandra, D.J. Williams, S.M. Howdle, K.M. Shakesheff, Control of pore size and structure of tissue engineering scaffolds produced by supercritical fluid processing, Eur. Cells Mater. 14 (2007) 64-77.

[21] A. Salerno, J. Saurina, C. Domingo, Supercritical CO2 foamed polycaprolactone scaffolds for controlled delivery of 5-fluorouracil, nicotinamide and triflusal, Int. J. Pharm. 496 (2015) 654-663.

[22] I. Tsivintzelis, G. Sanxaridou, E. Pavlidou, C. Panayiotou, Foaming of polymers with supercritical fluids: a thermodynamic investigation, J. Supercrit. Fluids 110 (2016) 
$240-250$.

[23] C. Ji, N. Annabi, A. Khademhosseini, F. Dehghani, Fabrication of porous chitosan scaffolds for soft tissue engineering using dense gas $\mathrm{CO}_{2}$, Acta Biomater. 7 (2011) 1653-1664.

[24] C. Tsioptsias, M.K. Paraskevopoulos, D. Christofilos, P. Andrieux, C. Panayiotou, Polymeric hydrogels and supercritical fluids: the mechanism of hydrogel foaming, Polymer 52 (2011) 2819-2826.

[25] N. Annabi, S.M. Mithieux, A.S. Weiss, F. Dehghani, The fabrication of elastin-based hydrogels using high pressure $\mathrm{CO}_{2}$, Biomaterials 30 (2009) 1-7.

[26] C. Tsioptsias, C. Panayiotou, Foaming of chitin hydrogels processed by supercritical carbon dioxide, J. Supercrit. Fluids 47 (2008) 302-308.

[27] Y.-B. Shim, H.-H. Jung, J.W. Jang, H.S. Yang, H. Bae, J.-C. Park, B. Choi, S.-H. Lee, Fabrication of hollow porous PLGA microspheres using sucrose for controlled dual delivery of dexamethasone and BMP2, J. Ind. Eng. Chem. 37 (2016) 101-106.

[28] P.F. Costa, A.M. Puga, L. Díaz-Gomez, A. Concheiro, D.H. Busch, C. AlvarezLorenzo, Additive manufacturing of scaffolds with dexamethasone controlled release for enhanced bone regeneration, Int. J. Pharm. 496 (2015) 541-550.

[29] P. Costa, J.M. Sousa Lobo, Modeling and comparison of dissolution profiles, Eur. J. Pharm. Sci. 13 (2001) 123-133.

[30] N.A. Peppas, J.J. Sahlin, A simple equation for the description of solute release. III. Coupling of diffusion and relaxation, Int. J. Pharm. 57 (1989) 169-172.

[31] V. Karageorgiou, D. Kaplan, Porosity of 3D biomaterial scaffolds and osteogenesis, Biomaterials 26 (2005) 5474-5491.

[32] A. Bamberger, G. Sieder, G. Maurer, High-pressure (vapor+liquid) equilibrium in binary mixtures of (carbon dioxide + water or acetic acid) at temperatures from 313 to 353 K, J. Supercrit. Fluids 17 (2000) 97-110.

[33] S. Wu, X. Liu, K.W.K. Yeung, C. Liu, X. Yang, Biomimetic porous scaffolds for bone tissue engineering, Mater. Sci. Eng. R 80 (2014) 1-36.

[34] V. Colombo, M. Čadová, L.M. Gallo, Mechanical behavior of bovine nasal cartilage under static and dynamic loading, J. Biomech. 46 (2013) 2137-2144.

[35] A. Arora, A. Kothari, D.S. Katti, Pore orientation mediated control of mechanical behavior of scaffolds and its application in cartilage-mimetic scaffold design, J. Mech. Behav. Biomed. Mater. 51 (2015) 169-183.

[36] N. Annabi, S.M. Mithieux, A.S. Weiss, F. Dehghani, Cross-linked open-pore elastic hydrogels based on tropoelastin, elastin and high pressure $\mathrm{CO}_{2}$, Biomaterials 31 (2010) 1655-1665.

[37] A. Gantar, L.P. da Silva, J.M. Oliveira, A.P. Marques, V.M. Correlo, S. Novak, R.L. Reis, Nanoparticulate bioactive-glass-reinforced gellan-gum hydrogels for bone-tissue engineering, Mater. Sci. Eng. C 43 (2014) 27-36.

[38] K. Rezwan, Q.Z. Chen, J.J. Blaker, A.R. Boccaccini, Biodegradable and bioactive porous polymer/inorganic composite scaffolds for bone tissue engineering, Biomaterials 27 (2006) 3413-3431.

[39] A.R.C. Duarte, J.F. Mano, R.L. Reis, Dexamethasone-loaded scaffolds prepared by supercritical-assisted phase inversion, Acta Biomater. 5 (2009) 2054-2062.

[40] A. Martins, A.R.C. Duarte, S. Faria, A.P. Marques, R.L. Reis, N.M. Neves, Osteogenic induction of hBMSCs by electrospun scaffolds with dexamethasone release functionality, Biomaterials 31 (2010) 5875-5885.

[41] A.R.C. Duarte, J.F. Mano, R.L. Reis, Preparation of chitosan scaffolds loaded with dexamethasone for tissue engineering applications using supercritical fluid technology, Eur. Polym. J. 45 (2009) 141-148.

[42] M.A. Kalam, Development of chitosan nanoparticles coated with hyaluronic acid for topical ocular delivery of dexamethasone, Int. J. Biol. Macromol. 89 (2016) 127-136. 